\title{
MicroRNAs: their involvement in fibrosis pathogenesis and use as diagnostic biomarkers in scleroderma
}

\author{
Honglin $\mathrm{Zhu}^{1}$, Hui $\mathrm{Luo}^{1}$ and Xiaoxia Zuo \\ miRNAs are important post-transcriptional regulators. The aberrant expression of miRNAs is strongly associated with the \\ initiation and progression of pathophysiologic processes in a wide range of human diseases. Scleroderma (systemic sclerosis; \\ $\mathrm{SSc}$ ) is a highly heterogeneous autoimmune disease that includes the progressive fibrotic replacement of normal tissue \\ architecture in multiple organs. Our previous studies have suggested that SSc skin tissues display a different miRNA expression \\ signature than that found in normal controls. miRNAs with pro- or antifibrotic properties are found to be dysregulated in SSc \\ skin fibrosis. Serum miRNA levels are associated with SSc activity and severity. miRNAs have the potential to be therapeutic \\ targets and serve as biomarkers for SSc diagnosis and assessment of disease state and severity. This review summarizes the \\ SSc miRNA expression signature and the roles of dysregulation of miRNAs in SSc tissues and serum and examines the future \\ therapeutic potential of targeting miRNAs in the management of SSc patients.
}

Experimental \& Molecular Medicine (2013) 45, e41; doi:10.1038/emm.2013.71; published online 20 September 2013

Keywords: biomarkers; fibrosis; microRNA; scleroderma

\section{INTRODUCTION}

miRNAs are a class of endogenous and evolutionarily conserved noncoding small RNAs (approximately 22 nucleotides) that bind to the $3^{\prime}$ untranslated regions of target genes. Once bound, miRNAs repress target gene translation and/or induce the degradation of the target gene mRNA. ${ }^{1}$ miRNAs are ubiquitously expressed in a wide range of species, including viruses, worms, flies, plants and animals. ${ }^{2,3}$ It is estimated that 1000 miRNAs are encoded in the human genome and expressed in a tissue- and cell type-specific manner. An individual miRNA can target numerous other mRNAs and can itself be targeted by multiple miRNAs, resulting in a vast regulatory potential. ${ }^{4}$ miRNAs have been shown to have fundamental roles in diverse biological and pathological processes, including intracellular signaling pathways, organ morphogenesis and disease processes. ${ }^{5}$ The aberrant expression of miRNAs is closely associated with the initiation and progression of pathophysiologic processes. ${ }^{4}$ It has been shown that extracellular miRNAs circulating in the blood can be measured. Most circulating miRNAs are found in lipid or lipoprotein complexes, such as apoptotic bodies, microvesicles, exosomes and other extracellular vesicles, resulting in a high degree of miRNA stability despite the presence of ribonucleases in both plasma and serum. ${ }^{6}$ The existence of miRNAs circulating in the blood has raised the possibility that miRNAs may possess the capability to serve as novel biomarkers. Recent reports have demonstrated that miRNAs represent potential biomarkers with distinct clinical characteristics, such as disease diagnosis or activity. ${ }^{7}$

Scleroderma (systemic sclerosis, SSc) is a complex heterogeneous autoimmune disease of unknown etiopathogenesis. SSc is characterized by vascular dysfunction and the excessive accumulation of the extracellular matrix (ECM), resulting in the progressive fibrotic replacement of normal tissue architecture and leading to the failure of affected organs, such as the kidney, heart and lung. ${ }^{8}$ The exact cellular and molecular mechanism of SSc remains unclear, but it involves complex interactions among vascular, immune and fibrotic processes in association with a genetic predisposition. ${ }^{9}$ To date, no therapy has been shown to reverse or arrest the progression of fibrosis, representing a major unmet medical need.

The selective targeting of the molecules and pathways that are involved in fibroblast activation, either singly or in combination, may offer new approaches in the treatment of

Department of Rheumatology, Xiangya Hospital, Central South University, Changsha, People's Republic of China

${ }^{1}$ These authors contributed equally to this work.

Correspondence: Dr X Zuo, Department of Rheumatology, Xiangya Hospital, Central South University, 87 Xiangya Road, Changsha, Hunan 410008, People's Republic of China.

E-mail: susanzuo@hotmail.com

Received 12 February 2013; revised 8 May 2013; accepted 20 May 2013 
fibrosis. The elevated expression of profibrotic miRNAs and/or reduced expression of antifibrotic miRNAs are likely to be important factors in the development of fibrosis in SSc patients. The regulation of miRNA expression could be therapeutically exploited to offer new treatments for SSc, and miRNAs circulating in the blood represent potential biomarkers for fibrosis.

In this review, we summarize recent advances in identifying the specific miRNAs involved in SSc pathogenesis, underscored by the potential use of specific miRNAs as novel biomarkers and therapeutic targets for the future management of SSc patients.

\section{miRNA EXPRESSION SIGNATURE IN SSc FIBROSIS}

miRNA expression profiles have been successfully utilized in the classification of human cancer, heart disease and autoimmune diseases. Studies of miRNA expression profiles in patients with SSc are expected to result in similar findings, revealing a biological and clinical relevance between miRNA expression and SSc disease state and severity. In our previous study, we identified the unique miRNA expression signature present in SSc skin samples. The two major subgroups of SSc, diffuse cutaneous scleroderma (dSSc) and limited cutaneous scleroderma (ISSc), displayed different miRNA expression signatures. We identified 42 miRNAs that were differentially expressed in dSSc and 60 miRNAs with altered expression in ISSc. A total of 21 miRNAs displaying altered expression in SSc skin samples were common between dSSc and ISSc. Among these miRNAs with changed expression, many were predicted to function as inhibitors of numerous mRNAs involved in autoimmune, vascular and fibrotic processes. ${ }^{10}$

Using miRNA microarray analysis, Li et al. ${ }^{11}$ identified 24 differentially expressed miRNAs from patients with SSc, including 9 upregulated miRNAs and 15 downregulated miRNAs. Through bioinformatics analysis and literature retrieval, they identified the target genes regulated by six of the miRNAs whose differential expression was correlated with SSc pathogenesis and hypothesized that the abnormal expression of some miRNAs may be important molecular events during the process of SSc genesis, development and evolution. ${ }^{11}$ The overlap between the miRNA expression signature identified in our study, which examined patients with SSc, and the signature identified in Li's study is relatively limited. In our study, we used skin samples from four patients with dSSc, two patients with ISSc, and three healthy volunteers as normal controls for miRNA array analysis. In Li's study, skin samples from three patients with SSc (the authors did not mention the SSc subgroups) and three healthy control volunteers were used for miRNA array analysis. The difference between the two studies can be explained by diversity in the disease severity, medical history and ethnicity of SSc patients, miRNA profiles of specific cell-types, and even more specifically, the developmental and functional organ status. ${ }^{12}$ Both our and Li's study revealed the unique miRNA expression signature in SSc patients, demonstrating that many of the dysregulation miRNAs exerted pro- or antifibrotic effects and participated in fibrosis.

\section{miRNAs DYSREGULATED IN SSc FIBROSIS}

Immune perturbations and vascular injury precede and contribute to the development of fibrosis in SSc, which, in turn, further exacerbate vascular and immune damage. ${ }^{9}$ Tissue fibrosis is the hallmark of SSc and is responsible for most SSc clinical manifestations. Microarray and real-time PCR analysis has shown that approximately 40 miRNAs are linked to fibrosis in various organs and disease settings. Most of these miRNAs are regulated by transforming growth factor (TGF) $-\beta$, which has been considered a crucial participant in the pathogenesis of the SSc fibrotic process. Many miRNAs have been shown to participate in repairing and remodeling the matrix including collagens, matrix metalloproteinases and integrins. miR-133, miR-141, miR-200a/b, miR-21 and miR-590 have been shown to directly induce or inhibit fibrosis by targeting the TGF- $\beta /$ Smad canonical signaling pathway. ${ }^{13-18}$ An analysis of miR-132, miR-133 and the miR-17-92 cluster (miR-18a, $19 \mathrm{a} / \mathrm{b}$ ) revealed that their targets were connective tissue growth factor. ${ }^{19-22} \mathrm{miR}-29 \mathrm{a} / \mathrm{b} / \mathrm{c}$, miR-377 (clustered with miR-382) and miR-449a/b have shown to be involved with ECM structural proteins or enzymes active in ECM remodeling. ${ }^{23-29}$ let7, miR-132, miR-155, miR-192 and miR-382 have been implicated in indirectly regulating fibrogenesis by affecting the epithelial-to-mesenchymal transition ${ }^{19,30-36}$ while miR-132, miR-15b, miR-16, miR-150, miR-27a, miR-27b, miR-335 and miR-34a were found to induce myofibroblast proliferation and resistance to apoptosis. ${ }^{19,37-42}$

\section{miR-29}

As described above, many miRNAs are involved in fibrosis. Among these, miR-29 has been extensively studied and is the best characterized direct regulator of ECM synthesis. ${ }^{43,44}$ miR29 exerts antifibrotic effects in several major fibrotic disorders, including SSc. miR-29 is known to directly repress the expression of collagen I, collagen III and collagen IV. Platelet-derived growth factor-B and TGF- $\beta$ can downregulate miR-29a expression, leading to the further upregulation of plateletderived growth factor-B and TGF- $\beta$. This positive feedback loop can lead to the uncontrolled accumulation of ECM proteins. ${ }^{44}$ Evidence has suggested that a strong downregulation of miR-29 expression in tissues and cells can cause a fibrotic response, including cardiac, pulmonary and liver fibrosis.

miR-29 was shown to negatively regulate COL1A1, COL1A2, COL3A1, FBN1 and ELN1 gene expression and to be responsible for ECM synthesis in myocardial infarction..$^{24}$ In lung fibrosis, miR-29 targeted many fibrosis-associated genes, including both structural ECM genes and enzymes involved in tissue remodeling. miR-29 levels were inversely correlated with the severity of fibrosis. ${ }^{25}$ In carbon tetrachloride-induced hepatic fibrogenesis mice, the miR-29 family was found to be significantly decreased in livers. The overexpression of miR-29b in a mouse model resulted in protection against 
salt-induced hypertensive renal medullary fibrosis after a highsalt diet. The silencing of miR-29b in the kidney of these animals caused the upregulation of a large number of ECM and ECM-modulating genes. ${ }^{26}$

Studies investigating the role of miR-29 in SSc are ongoing. Our previous study demonstrated that miR-29 was decreased in both SSc skin tissues and primary fibroblasts. The downregulation of miR-29 was correlated with the upregulation of COL1A1 mRNA. ${ }^{10}$ Maurer et al. ${ }^{23}$ identified miR-29 as a key regulator of collagen expression in SSc. In their study, miR-29 was downregulated in cultured dermal fibroblasts derived from SSc skin, resulting in excessive collagen production. In a bleomycin-induced mouse model of skin fibrosis, the tyrosinekinase inhibitor imatinib mesylate was shown to inhibit the platelet-derived growth factor-B and TGF- $\beta$ pathways and restore miR-29a levels. The supplementation of miR-29a decreased collagen expression in SSc fibroblasts. In addition, serum miR-29a levels were found to be significantly decreased in the very early stage of SSc. ${ }^{45}$

All of these studies suggest that miR-29 expression was downregulated by TGF- $\beta$ and that decreased miR-29 expression was involved in SSc tissue fibrosis. Other signaling pathways also involve miR-29, such as the Wnt/b-catenin, nuclear factor-kappaB (NF- $\mathrm{\kappa B})$ and mitogen-activated protein kinases pathways. ${ }^{43}$ miR-29 could participate in other biological pathways involved in the fibrosis process.

\section{OTHER miRNAs}

In addition to miR-29, there are many other miRNAs known to undergo SSc fibrosis regulation, (Table 1). Our preliminary data showed that miR-21 was increased and that miR-145 was decreased in both SSc skin tissues and fibroblasts. After stimulation with TGF- $\beta$, the expression of miR-21 was increased and that of Smad7 mRNA was decreased. miR-145 was also upregulated, whereas $\operatorname{Smad} 3$ mRNA levels were downregulated. miR-21 and miR-145 may exert pro- or antifibrosis effects in SSc. ${ }^{10}$ Further, we studied the direct one-toone relationship between miR-21 and Smad7. We found that Smad7 was a direct target of miR-21 and that miR-21 was induced by TGF- $\beta$, which in turn promoted the TGF- $\beta$ induced fibrogenic activation of skin fibroblasts through the targeting of Smad7 (data not shown). Honda et al. ${ }^{46}$ demonstrated decreased miR-150 expression in SSc fibroblasts both in vivo and in vitro. The transfection of a miR-
150 inhibitor into normal fibroblasts induced the expression of integrin $\beta 3$, phosphorylated Smad3 and COL1A1, whereas the forced overexpression of miR-150 resulted in the downregulation of these genes in SSc fibroblasts. miR-150 was shown to have an important role in the pathogenesis of SSc. miR-196a directly contributed to the constitutive upregulation of COL1A1 and COL1A2 expression in SSc fibroblasts. The DDR2-miR-196a pathway was a negative feedback system and impairment of this pathway may be involved in the pathogenesis of SSc. ${ }^{47}$ The inhibition of miR-196a led to the overexpression of COL1A1 and COL1A2 in normal fibroblasts, whereas the overexpression of miR-196a resulted in the downregulation of COL1A1 and COL1A2 in SSc fibroblasts. ${ }^{48}$ Compared with untreated normal fibroblasts, miR-92a expression was significantly increased in TGF- $\beta$ treated normal cultured dermal fibroblasts and SSc fibroblasts. The overexpression of miR-92a in normal fibroblasts resulted in the downregulation of MMP-1 expression. ${ }^{49}$

The miRNAs described above exert pro- or antifibrotic effects in SSc through different signaling pathways. They may be common regulators of fibrosis in different organs and disease settings. Inhibiting the function of these miRNAs through the use of anti-miR oligonucleotides, small-molecule inhibitors, miRNA sponges and miRNA masks/target protectors and promoting the activity of antifibrotic miRNAs through gene therapy or delivery of miRNA mimics can serve as novel therapeutic options in the treatment of fibrosis.

\section{MIRNA AS BIOMARKERS IN SSC}

Fibrosis is the most important and prominent clinical feature of SSc and occurs in multiple organs. The extent and severity of tissue fibrosis correlates with prognosis and mortality in SSc. However, there is an unmet need for reliable and accurate biomarkers that reflect the fibrotic process in SSc. Serum miRNA levels can be used as valuable biomarkers for the diagnosis, prognosis and therapeutic value of various diseases. The expression pattern of miRNAs is reflective of the underlying pathophysiologic processes specific to various disease states. Disease-associated miRNAs can be measured from various sources, such as blood components, biologic fluids and tissue samples. ${ }^{50}$ miRNAs are highly stable and appear to be resistant to variations in sample handling (they can even be detected in formalin-fixed paraffin-embedded samples by microarray profiling or in situ hybridization), which increase

Table 1 miRNAs linked to SSc fibrosis

\begin{tabular}{llllr}
\hline miRNA & Effect & Tissue examined & Target gene and protein \\
\hline miR-29 & Antifibrosis & SSc fibroblasts, bleomycin-induced & COL1A1, COL1A2, COL3A1, FBN1, \\
& & Skin fibrosis & ELN1, ECM synthesis & 10, 23-26, 43-45 \\
miR-21 & Profibrosis & SSc skin tissues, SSc fibroblasts & Smad7 & 10 \\
miR-150 & Antifibrosis & SSc fibroblasts & Integrin- 33 & 46 \\
miR-196a & Antifibrosis & SSc fibroblasts & COL1A1, COL1A2 & 47,48 \\
miR-92a & Profibrosis & SSc fibroblasts & MMP-1 & 49
\end{tabular}

Abbreviations: ECM, extracellular matrix; miRNA, microRNA; SSc, systemic sclerosis. 
their appeal as practical biomarkers. ${ }^{50}$ Good correlations between serum and tissue miRNA profiles have already been shown for several cancers and various other diseases. Reports suggest that miRNAs have important roles in systemic rheumatic diseases. Various diseases and different stages of the same disease are associated with distinct miRNA expression profiles. ${ }^{7}$ The biomarker potential of many serum miRNAs has been investigated in patients with SSc. The elevated expression of profibrotic miRNAs and/or reduced expression of antifibrotic miRNAs are likely to be important factors in the development of fibrosis in SSc. Circulating miRNAs are promising biomarker candidates for the diagnosis, prognosis and assessment of disease activity and severity (Table 2).

Serum miR-150 levels were found to be decreased in SSc patients, and SSc patients with lower serum miR-150 levels had more severe clinical manifestations. ${ }^{46}$ Patients with lower serum miR-196a levels had a significantly higher ratio of dSSc:ISSc, a higher modified Rodnan total skin thickness score, and a higher prevalence of pitting scars than those with higher miR-196a levels. ${ }^{48}$ Serum levels of miR-92a were significantly higher in SSc patients than in normal subjects, and patients with increased miR-92a levels tended to have telangiectasia at a lower frequency than those with normal levels. ${ }^{49}$ Makino et al. ${ }^{51}$ collected serum samples from 61 patients with SSc, 8 patients with systemic lupus erythematosus, 8 patients with dermatomyositis, 12 patients with scleroderma spectrum disorder and 20 healthy controls. Analysis of the samples by realtime PCR indicated that the miR-142-3p levels in patients with SSc were significantly higher than those in patients with systemic lupus erythematosus, dermatomyositis, scleroderma spectrum disorder (SSD) and healthy control subjects. The serum levels of miR-142-3p were correlated with the severity of SSc fibrosis and may be useful diagnostic markers for the presence of SSc and differentiation of SSc from scleroderma spectrum disorder.

COL1A1 is a target of miR-29a and is upregulated in SSc patients. Kawashita et al. ${ }^{45}$ also determined miR-29a levels in serum samples from 61 SSc patients. Serum miR-29a levels were not downregulated in SSc, and there was no statistically significant difference between healthy control subjects and SSc patients. However, SSc patients with reduced miR-29a levels had significantly higher right ventricular systolic pressure by
Doppler echocardiography than those with normal miR-29a levels. Although the cause of pulmonary hypertension in SSc is still uncertain, this result suggested that miR-29a also has a role in the pathogenesis of pulmonary hypertension. ${ }^{45}$

\section{CONCLUSIONS}

miRNAs are important post-transcriptional regulators that may repress more than $60 \%$ of all mammalian protein-coding genes. ${ }^{52,53}$ They are strongly associated with the pathogenesis of a wide range of human diseases. Disease-associated miRNAs represent a new class of targets for the development of miRNA-based therapeutic modalities, which may yield patient benefits that are unobtainable using other therapeutic approaches. As outlined in this review, there is increasing evidence that miRNAs have a role in the regulation of fibrosis in SSc patients. Similar to antisense molecules that have been used therapeutically for fibrosis, the use of miRNA as a therapeutic target provides theoretical advantages over the current drug design strategies in SSc fibrosis, which are focused on single-gene targeting. The specific inhibition of a miRNA or the addition of a single miRNA mimetic may produce a phenotype that is derived from a complex set of gene expression changes. Clinical results with miR-122 inhibition in HCV patients validate the concept that miRNA modulation in humans is possible. ${ }^{54}$ Although the field of miRNA therapeutics is in its infancy, promising data in both laboratory animals and in humans already exist. ${ }^{55}$

Because miRNAs exhibit remarkable stability, as well as an ease and reliability of detection, miRNAs from blood samples or urine samples of patients hold promise as novel noninvasive clinical biomarkers, especially for early diagnosis. ${ }^{56}$ Provocative ideas on miRNA and its role in the pathogenesis of diverse autoimmune diseases including SSc have been put forward. The identification of specific miRNAs as biomarkers of disease severity will pave the way for the development of disease- and stage-specific targeted therapies.

\section{REVIEW CRITERIA}

A search for original articles published between 2007 and 2013 was performed using the PubMed database. The search terms used were 'microRNA,' 'fibrosis,' 'SSc or scleroderma or systemic sclerosis' and 'precursor and process', alone and in combination. All articles identified were full-text English-

Table 2 miRNAs as biomarkers in SSc

\begin{tabular}{lll}
\hline miRNA in serum & Expression changes & Effect \\
\hline miR-29a & Decreased & Higher right ventricular systolic pressure \\
miR-150 & Decreased & More severe clinical manifestations \\
miR-196a & Decreased & Higher ratio of dssc:Issc, higher modified Rodnan total skin thickness score, \\
miR-92a & higher prevalence of pitting scars \\
miR-142-3p & A lower frequency of telangiectasia \\
& Increased & $\begin{array}{l}\text { Diagnostic markers for differentiation of SSc from SSD, early detection of } \\
\text { developing SSc, a short sublingual frenulum }\end{array}$ \\
\hline
\end{tabular}

Abbreviations: miRNA, microRNA; SSc, systemic sclerosis; SSD, scleroderma spectrum disorder. 
language papers. Papers cited in this review were selected based on the authors' view of their direct relevance to the current topic.

\section{CONFLICT OF INTEREST}

The authors declare no conflict of interest.

1 O'Connell RM, Rao DS, Chaudhuri AA, Baltimore D. Physiological and pathological roles for microRNAs in the immune system. Nat Rev Immunol 2010; 10: 111-122.

2 Bartel DP. MicroRNAs: genomics, biogenesis, mechanism, and function. Cell 2004; 116: 281-297.

3 Carrington JC, Ambros V. Role of microRNAs in plant and animal development. Science 2003; 301: 336-338.

4 Bushati N, Cohen SM. microRNA functions. Annu Rev Cell Dev Biol 2007; 23: 175-205.

5 Stefani G, Slack FJ. Small non-coding RNAs in animal development. Nat Rev Mol Cell Biol 2008; 9: 219-230.

6 Kosaka N, Iguchi H, Ochiya T. Circulating microRNA in body fluid: a new potential biomarker for cancer diagnosis and prognosis. Cancer Sci 2010; 101: 2087-2092.

7 Alevizos I, Illei GG. MicroRNAs as biomarkers in rheumatic diseases. Nat Rev Rheumatol 2010; 6: 391-398.

8 Gabrielli A, Avvedimento EV, Krieg T. Scleroderma. N Engl J Med 2009; 360: 1989-2003.

9 Bhattacharyya S, Wei J, Varga J. Understanding fibrosis in systemic sclerosis: shifting paradigms, emerging opportunities. Nat Rev Rheumatol 2012; 8: 42-54.

10 Zhu H, Li Y, Qu S, Luo H, Zhou Y, Wang Y et al. MicroRNA expression abnormalities in limited cutaneous scleroderma and diffuse cutaneous scleroderma. J Clin Immunol 2012; 32: 514-522.

$11 \mathrm{Li} \mathrm{H}$, Yang R, Fan X, Gu T, Zhao Z, Chang D et al. MicroRNA array analysis of microRNAs related to systemic scleroderma. Rheumatol Int 2012; 32: 307-313.

12 Neilson JR, Zheng GX, Burge CB, Sharp PA. Dynamic regulation of miRNA expression in ordered stages of cellular development. Genes Dev 2007; 21: 578-589.

13 Shan H, Zhang Y, Lu Y, Pan Z, Cai B, Wang N et al. Downregulation of miR-133 and miR-590 contributes to nicotine-induced atrial remodelling in canines. Cardiovasc Res 2009; 83: 465-472.

14 Wang B, Koh P, Winbanks C, Coughlan MT, McClelland A, Watson A et al. miR-200a prevents renal fibrogenesis through repression of TGF-beta2 expression. Diabetes 2011; 60: 280-287.

15 Liu G, Friggeri A, Yang Y, Milosevic J, Ding Q, Thannickal VJ et al. miR-21 mediates fibrogenic activation of pulmonary fibroblasts and lung fibrosis. J Exp Med 2010; 207: 1589-1597.

16 Thum T, Gross C, Fiedler J, Fischer T, Kissler S, Bussen M et al. MicroRNA-21 contributes to myocardial disease by stimulating MAP kinase signalling in fibroblasts. Nature 2008; 456: 980-984.

17 Zhong X, Chung AC, Chen HY, Meng XM, Lan HY. Smad3-mediated upregulation of miR-21 promotes renal fibrosis. J Am Soc Nephrol 2011; 22: 1668-1681.

18 Kato M, Putta S, Wang M, Yuan H, Lanting L, Nair I et al. TGF-beta activates Akt kinase through a microRNA-dependent amplifying circuit targeting PTEN. Nat Cell Biol 2009; 11: 881-889.

19 Mann J, Chu DC, Maxwell A, Oakley F, Zhu NL, Tsukamoto H et al. MeCP2 controls an epigenetic pathway that promotes myofibroblast transdifferentiation and fibrosis. Gastroenterology 2010; 138: 705-714.

20 Duisters RF, Tijsen AJ, Schroen B, Leenders JJ, Lentink V, van der Made I et al. miR-133 and miR-30 regulate connective tissue growth factor: implications for a role of microRNAs in myocardial matrix remodeling. Circ Res 2009; 104: 170-178.

21 Kodama T, Takehara T, Hikita H, Shimizu S, Shigekawa M, Tsunematsu H et al. Increases in p53 expression induce CTGF synthesis by mouse and human hepatocytes and result in liver fibrosis in mice. J Clin Invest 2011; 121: 3343-3356.

22 van Almen GC, Verhesen W, van Leeuwen RE, van de Vrie M, Eurlings $C$, Schellings MW et al. . MicroRNA-18 and microRNA-19 regulate CTGF and TSP-1 expression in age-related heart failure. Aging Cell 2011; 10: 769-779.
23 Maurer B, Stanczyk J, Jungel A, Akhmetshina A, Trenkmann M, Brock M et al. MicroRNA-29, a key regulator of collagen expression in systemic sclerosis. Arthritis Rheum 2010; 62: 1733-1743.

24 van Rooij E, Sutherland LB, Thatcher JE, DiMaio JM, Naseem RH, Marshall WS et al. Dysregulation of microRNAs after myocardial infarction reveals a role of miR-29 in cardiac fibrosis. Proc Natl Acad Sci USA 2008; 105: 13027-13032.

25 Cushing L, Kuang PP, Qian J, Shao F, Wu J, Little F et al. miR-29 is a major regulator of genes associated with pulmonary fibrosis. Am J Respir Cell Mol Biol 2011; 45: 287-294.

26 Liu Y, Taylor NE, Lu L, Usa K, Cowley AW Jr., Ferreri NR et al. Renal medullary microRNAs in Dahl salt-sensitive rats: miR-29b regulates several collagens and related genes. Hypertension 2010; 55: 974-982.

27 Du B, Ma LM, Huang MB, Zhou H, Huang HL, Shao P et al. High glucose down-regulates miR-29a to increase collagen IV production in HK-2 cells. FEBS Lett 2010; 584: 811-816.

28 Wang Q, Wang Y, Minto AW, Wang J, Shi Q, Li X et al. MicroRNA-377 is upregulated and can lead to increased fibronectin production in diabetic nephropathy. FASEB J 2008; 22: 4126-4135.

29 Muth M, Theophile K, Hussein K, Jacobi C, Kreipe H, Bock O. "Hypoxiainduced down-regulation of microRNA-449a/b impairs control over targeted SERPINE1 (PAI-1) mRNA - a mechanism involved in SERPINE1 (PAI-1) overexpression". J Trans/ Med 2010; 8: 33.

30 Pandit KV, Corcoran D, Yousef H, Yarlagadda M, Tzouvelekis A, Gibson KF et al. Inhibition and role of let-7d in idiopathic pulmonary fibrosis. Am J Respir Crit Care Med 2010; 182: 220-229.

31 Katare R, Riu F, Mitchell K, Gubernator M, Campagnolo P, Cui Y et al. Transplantation of human pericyte progenitor cells improves the repair of infarcted heart through activation of an angiogenic program involving micro-RNA-132. Circ Res 2011; 109: 894-906.

32 Pottier N, Maurin T, Chevalier B, Puissegur MP, Lebrigand K, RobbeSermesant $\mathrm{K}$ et al. Identification of keratinocyte growth factor as a target of microRNA-155 in lung fibroblasts: implication in epithelial-mesenchymal interactions. PLoS One 2009; 4: e6718.

33 Kato M, Zhang J, Wang M, Lanting L, Yuan H, Rossi JJ et al. MicroRNA192 in diabetic kidney glomeruli and its function in TGF-beta-induced collagen expression via inhibition of E-box repressors. Proc Natl Acad Sci USA 2007; 104: 3432-3437.

34 Wang B, Herman-Edelstein M, Koh P, Burns W, Jandeleit-Dahm K, Watson A et al. E-cadherin expression is regulated by miR-192/215 by a mechanism that is independent of the profibrotic effects of transforming growth factor-beta. Diabetes 2010; 59: 1794-1802.

35 Oba S, Kumano S, Suzuki E, Nishimatsu H, Takahashi M, Takamori H et al. miR-200b precursor can ameliorate renal tubulointerstitial fibrosis. PLoS One 2010; 5: e13614.

36 Kriegel AJ, Fang Y, Liu Y, Tian Z, Mladinov D, Matus IR et al. MicroRNAtarget pairs in human renal epithelial cells treated with transforming growth factor beta 1: a novel role of miR-382. Nucleic Acids Res 2010; 38: 8338-8347.

37 Guo CJ, Pan Q, Li DG, Sun H, Liu BW. miR-15b and miR-16 are implicated in activation of the rat hepatic stellate cell: An essential role for apoptosis. J Hepatol 2009; 50: 766-778.

38 Ezzie ME, Crawford M, Cho JH, Orellana R, Zhang S, Gelinas R et al. Gene expression networks in COPD: microRNA and mRNA regulation. Thorax 2012; 67: 122-131.

39 Venugopal SK, Jiang J, Kim TH, Li Y, Wang SS, Torok NJ et al. Liver fibrosis causes downregulation of miRNA-150 and miRNA-194 in hepatic stellate cells, and their overexpression causes decreased stellate cell activation. Am J Physiol Gastrointest Liver Physiol 2010; 298: G101-G106.

$40 \mathrm{Ji}$ J, Zhang J, Huang G, Qian J, Wang X, Mei S. Over-expressed microRNA$27 \mathrm{a}$ and $27 \mathrm{~b}$ influence fat accumulation and cell proliferation during rat hepatic stellate cell activation. FEBS Lett 2009; 583: 759-766.

41 Chen C, Wu CQ, Zhang ZQ, Yao DK, Zhu L. Loss of expression of miR-335 is implicated in hepatic stellate cell migration and activation. Exp Cell Res 2011; 317: 1714-1725.

42 Li WQ, Chen C, Xu MD, Guo J, Li YM, Xia QM et al. The rno-miR-34 family is upregulated and targets ACSL1 in dimethylnitrosamine-induced hepatic fibrosis in rats. FEBS J 2011; 278: 1522-1532.

43 Kriegel AJ, Liu Y, Fang Y, Ding X, Liang M. The miR-29 family: genomics, cell biology, and relevance to renal and cardiovascular injury. Physiol Genomics 2012; 44: 237-244. 
44 Peng WJ, Tao JH, Mei B, Chen B, Li BZ, Yang GJ et al. MicroRNA-29: a potential therapeutic target for systemic sclerosis. Expert Opin Ther Targets 2012; 16: 875-879.

45 Kawashita Y, Jinnin M, Makino T, Kajihara I, Makino K, Honda N et al. Circulating miR-29a levels in patients with scleroderma spectrum disorder. J Dermatol Sci 2011; 61: 67-69.

46 Honda N, Jinnin M, Kira-Etoh T, Makino K, Kajihara I, Makino T et al. miR150 down-regulation contributes to the constitutive type | collagen overexpression in scleroderma dermal fibroblasts via the induction of integrin beta3. Am J Pathol 2013; 182: 206-216.

47 Makino K, Jinnin M, Aoi J, Hirano A, Kajihara I, Makino T et al. Discoidin domain receptor 2-microRNA 196a-mediated negative feedback against excess type I collagen expression is impaired in scleroderma dermal fibroblasts. J Invest Dermatol 2013; 133: 110-119.

48 Honda N, Jinnin M, Kajihara I, Makino T, Makino K, Masuguchi S et al. TGF-beta-mediated downregulation of microRNA-196a contributes to the constitutive upregulated type I collagen expression in scleroderma dermal fibroblasts. J Immunol 2012; 188: 3323-3331.

49 Sing T, Jinnin M, Yamane K, Honda N, Makino K, Kajihara I et al. microRNA-92a expression in the sera and dermal fibroblasts increases in patients with scleroderma. Rheumatology (Oxford) 2012; 51: $1550-1556$.

50 Dai R, Ahmed SA. MicroRNA, a new paradigm for understanding immunoregulation, inflammation, and autoimmune diseases. Trans/ Res 2011; 157: 163-179.
51 Makino K, Jinnin M, Kajihara I, Honda N, Sakai K, Masuguchi S et al. Circulating miR-142-3p levels in patients with systemic sclerosis. Clin Exp Dermatol 2012; 37: 34-39.

52 Friedman LM, Avraham KB. MicroRNAs and epigenetic regulation in the mammalian inner ear: implications for deafness. Mamm Genome 2009; 20: 581-603.

53 Bartel DP. MicroRNAs: target recognition and regulatory functions. Cell 2009; 136: 215-233.

54 Janssen HL, Reesink HW, Zeuzem S, Lawitz E, Rodriguez-Torres M, Chen A et al. A randomized, double-blind, placebo (plb) controlled safety and antiviral proof of concept study of miravirsen (MIR), an oligonucleotide targeting miR-122, in treatment naïve patients with genotype 1 (gt1) chronic HCV infection [abstract]. Hepatology 2011; 54: 1430A.

55 Stenvang J, Petri A, Lindow M, Obad S, Kauppinen S. Inhibition of microRNA function by antimiR oligonucleotides. Silence 2012; 3: 1 .

56 Gilad S, Meiri E, Yogev Y, Benjamin S, Lebanony D, Yerushalmi N et al. Serum microRNAs are promising novel biomarkers. PLoS One 2008; 3: e3148.

(1) $\odot \odot$ This work is licensed under a Creative Commons Attribution-NonCommercial-NoDerivs 3.0 Unported License. To view a copy of this license, visit http:// creativecommons.org/licenses/by-nc-nd/3.0/ 